\title{
THE SECOND CONJUGATE ALGEBRA OF THE FOURIER ALGEBRA OF A LOCALLY COMPACT GROUP \\ BY
}

\author{
ANTHONY TO-MING LAU ${ }^{1}$
}

\begin{abstract}
Let $G$ be a locally compact group and let $V N(G)$ denote the von Neumann algebra generated by the left translations of $G$ on $L_{2}(G)$. Then $V N(G)^{*}$, when regarded as the second conjugate space of the Fourier algebra of $G$, is a Banach algebra with the Arens product. We prove among other things that when $G$ is amenable, $V N(G)^{*}$ is neither commutative nor semisimple unless $G$ is finite. We study in detail the class of maximal regular left ideals in $V N(G)^{*}$. We also show that if $G_{1}$ and $G_{2}$ are discrete groups, then $G_{1}$ and $G_{2}$ are isomorphic if and only if $V N\left(G_{1}\right)^{*}$ and $V N\left(G_{2}\right)^{*}$ are isometric order isomorphic.
\end{abstract}

1. Introduction. Let $G$ be a locally compact group. Then $A(G)$, the Fourier algebra of $G$, is the linear subspace of $C_{0}(G)$ (bounded continuous complex-valued functions on $G$ vanishing at infinity) consisting of all functions of the form $(h * \tilde{k})$ where $h, k \in L_{2}(G), \check{g}(x)=g\left(x^{-1}\right)$ and $\tilde{k}=k\left(x^{-1}\right)$. If $f \in L_{2}(G)$ and $g \in G$, define $\lambda(g) f(t)=f\left(g^{-1} t\right), t \in G$. Let $V N(G)$ denote the closure in the weak operator topology of the linear span of $\{\lambda(g) ; g \in G\}$ in $\mathscr{B}\left(L_{2}(G)\right)$, the algebra of bounded linear operators from $L_{2}(G)$ into $L_{2}(G)$. Then $\phi=(h * \tilde{k})^{\swarrow}$ in $A(G)$ can be regarded as an ultraweakly continuous linear functional on the von Neumann algebra $V N(G)$ defined by $\phi(T)=(T h, k)$ (inner product in $L_{2}(G)$ ) for each $T \in V N(G)$. Furthermore, each ultraweakly continuous linear functional on $V N(G)$ is of this form [6, pp. 210 and 218]. In particular, $A(G)$ can be identified as the unique predual of $V N(G)$. Also, $A(G)$ with pointwise multiplication and the norm $\|\phi\|=\sup \{|\phi(x)| ; x \in V N(G)$ and $\|x\| \leqslant 1\}$ is a commutative Banach algebra with spectrum $G$ [6, p. 222].

It is the purpose of this paper to study the Banach algebra $V N(G)^{*}$, the second conjugate algebra of $A(G)$ equipped with the Arens product [1]. We prove, in \$3, that $V N(G)^{*}$ is semisimple if and only if $G$ is finite. We also prove that $A(G)$ is an ideal in $V N(G)^{*}$ if and only if $G$ is discrete. When $G$ is amenable, then the Fourier-Stieltjes algebra of $G$ is a quotient of $V N(G)^{*}$. We characterize, in $\S 4$, the regular maximal left ideals in $V N(G)^{*}$. We show that any such ideal must contain $I_{g}=\{\phi \in A(G) ; \phi(g)=0\}$ for some $g \in G$. Furthermore, if $G$ is amenable, then every regular maximal left ideal in $V N(G)^{*}$ contains a unique $I_{g}, g \in G$, if and

Received by the editors August 7, 1980.

AMS (MOS) subject classifications (1970). Primary 43A30, 43A65; Secondary 43A07, 43A35, 46L25, 46H10.

Key words and phrases. Arens product, second conjugate algebra, Fourier algebra, Fourier-Stieltjes algebra, left regular representation, amenable locally compact group, regular ideal, group $C^{*}$-algebra, Tauberian property, radical, uniformly continuous functionals, topological invariant mean.

${ }^{1}$ This research is supported by NRC grant A-7679. 
only if $G$ is compact. We also show that $G$ is discrete if and only if each regular left ideal in $V N(G)^{*}$ either contains $A(G)$ or must be the kernel of some $\lambda(g) \in$ $V N(G), g \in G$. In $\S 5$ this result is used to prove the following isomorphism theorem for discrete groups: if $G_{1}$ and $G_{2}$ are discrete groups, then the Banach algebras $V N\left(G_{1}\right)^{*}$ and $V N\left(G_{2}\right)^{*}$ are isometric order isomorphic if and only if $G_{1}$ and $G_{2}$ are isomorphic.

Most of our results in $\S \S 3$ and 4 are known and proved by Civin and Yood [4] and Civin [3] when $G$ is an abelian locally compact group. In this case, $A(G)$ is isometric isomorphic to $L_{1}(\hat{G})$ via the Fourier transform. Our proofs depend heavily on some deep analysis of the Fourier algebra $A(G)$ and various subspaces of $V N(G)$ of P. Eymard [6] and E. Granirer [7]-[9].

2. Preliminaries. Let $E$ be a linear space, and $\phi$ be a linear functional on $E$, then the value of $\phi$ at an element $x$ in $E$ will be written as $\phi(x)$ or $\langle\phi, x\rangle$.

Throughout this paper, $G$ denotes a locally compact group with a fixed left Haar measure. Let $C(G)$ denote the Banach space of bounded continuous complexvalued functions on $G$ with the supremum norm. Then $G$ is amenable if there exists a positive linear functional $\phi$ on $C(G)$ of norm one such that $\phi\left({ }_{a} f\right)=\phi(f)$ for each $a \in G$ and each $f \in C(G)$, where ${ }_{a} f(t)=f(a t)$ for each $t \in G$. Amenable groups include all solvable groups and all compact groups. However, the free group on two generators is not amenable.

Let $P(G)$ denote the subspace of $C(G)$ consisting of all continuous positive definite functions on $G$, and let $B(G)$ be its linear span. Then $B(G)$ can be identified with the dual of $C^{*}(G)$, the group $C^{*}$-algebra of $G$ (see [6, p. 192]). Also, $B(G)$ with pointwise multiplication and the dual norm is a commutative Banach algebra called the Fourier-Stieltjes algebra of $G$ [6, Proposition 2.16].

Let $P_{\rho}(G)$ denote the closure of $P(G) \cap C_{00}(G)$ in the compact open topology, where $C_{00}(G)$ is the set of all functions in $C(G)$ with compact support, and let $B_{\rho}(G)$ denote the linear span of $P_{\rho}(G)$. Then $B_{\rho}(G)$ is a closed ideal in $B(G)$ and $B_{\rho}(G)$ is precisely the dual of $C_{\rho}^{*}(G)$ [6, Propositions 1.21 and 2.16], where $C_{\rho}^{*}(G)$ is the norm closure of $\left\{\lambda(f) ; f \in L_{1}(G)\right\}$ in $\mathscr{B}\left(L_{2}(G)\right)$, and $\lambda(f)(h)=f * h$ for $h \in L_{2}(G)$. As is known [10, p. 61], $B_{\rho}(G)=B(G)$ if and only if $G$ is amenable.

The Fourier algebra $A(G)$ as defined in $\S 1$ is the closed linear span of $P(G) \cap$ $C_{00}(G)$. It is a closed ideal in $B(G)$ (see [6, p. 208]). Also $A(G) \subseteq B_{\rho}(G)$.

There is a natural module action of $A(G)$ on $V N(G)$ given by $\langle\phi \cdot x, \gamma\rangle=$ $\langle\phi \gamma, x\rangle$ for each $\phi, \gamma \in A(G)$ and each $x \in V N(G)$. Recently E. Granirer [7, p. 373] has defined the subspace $U C B(\hat{G})$ of $V N(G)$ as the norm closure of $A(G)$. $V N(G)$. Then $U C B(\hat{G})$ is a $C^{*}$-subalgebra of $V N(G)$ [8, Proposition $\left.2\left(\mathrm{a}_{1}\right)\right]$ and elements in $U C B(\hat{G})$ are called uniformly continuous functionals on $A(G)$. It follows from [8, Propositions 1 and 3] and [9, Theorem 3] that $U C B(\hat{G})=V N(G)$ if and only if $G$ is compact.

A positive linear functional $m \in V N(G)^{*}$ of norm one is called a topological invariant mean on $V N(G)$ if $\langle m, \phi \cdot x\rangle=\langle m, x\rangle$ for each $x \in V N(G)$ and each $\phi \in P(G) \cap A(G)$ such that $\phi(e)=1$ where $e$ is the identity of $G$. As known [16, Theorem 4], $V N(G)$ always has a topological invariant mean. 
A subset $X$ of $V N(G)$ is topologically invariant if $\phi \cdot x \in X$ for each $\phi \in A(G)$ and each $x \in X$. If $X$ is a topological invariant linear subspace of $V N(G)$, we say that $X$ is topologically introverted if for each $m \in V N(G)^{*}$ and each $x \in X$, the functional $\gamma \rightarrow\langle m, \gamma \cdot x\rangle$ on $A(G)$, denoted by $m \odot x$, also defines an element in $X$. Examples of topologically introverted subspaces of $V N(G)$ include $U C B(\hat{G})$ and $C_{\rho}^{*}(G)$ (see [13, Proposition 5.2]). Also, any weak*-closed topologically invariant subspace of $V N(G)^{*}$ is topologically introverted [13, Lemma 5.1].

3. The Banach algebra $V N(G)^{*}$. In [1] Arens shows that given a Banach algebra $B$, it is possible to define a multiplication on $B^{* *}$ which extends multiplication on $B$. In case $B=A(G), m, n \in V N(G)^{*}$, the Arens product $m \odot n$ is defined by the formula: $\langle m \odot n, x\rangle=\langle m, n \odot x\rangle$ for each $x \in V N(G)$, where $\langle n \odot x, \phi\rangle=$ $\langle n, \phi \cdot x\rangle, \phi \in A(G)$. This same formula certainly makes sense when $V N(G)$ is replaced by a topologically invariant and introverted subspace $X$ of $V N(G)$. The following observations will be useful in the sequel.

LEMMA 3.1. (i) $A(G)$ is in the centre of $V N(G)^{*}$.

(ii) For each $n \in V N(G)^{*}$, the map $m \rightarrow m \odot n$ from $V N(G)^{*}$ into $V N(G)^{*}$ is weakly*-weak* continuous.

(iii) Any weak*-closed right ideal in $V N(G)^{*}$ is an ideal.

(iv) If $g \in G$, then the functional on $V N(G)^{*}$ defined by $m \rightarrow\langle m, \lambda(g)\rangle$ is multiplicative.

Proof. Both (ii) and (iii) are immediate. Also (iii) follows from (i) and [13, Lemma 5.1]. To prove (iv), we simply observe that if $n \in V N(G)^{*}$, then $n \odot \lambda(g)=$ $\langle n, \lambda(g)\rangle \lambda(g)$. Hence $\langle m \odot n, \lambda(g)\rangle=\langle m, \lambda(g)\rangle\langle n, \lambda(g)\rangle$ for any $m \in V N(G)^{*}$.

Proposition 3.2. (a) $V N(G)^{*}$ has a right identity if and only if $G$ is amenable.

(b) $V N(G)^{*}$ has a left identity if and only if $G$ is compact.

Proof. (a) is a direct consequence of Leptin's theorem [14] and Proposition 7 in [2, p. 146].

(b) If $G$ is compact, then $A(G)=B(G)$. Hence $A(G)$ has an identity which is also the identity for $V N(G)^{*}$. Conversely, if $G$ is not compact, then $U C B(\hat{G})$ is a proper $C^{*}$-subalgebra of $V N(G)$ by Granirer's results [8, Proposition 1] and [9, Theorem 3]. Hence there exists $m \in V N(G)^{*}$ such that $m \neq 0$ and $m(y)=0$ for all $y \in U C B(\hat{G})$. Then $m \odot x=0$ for each $x \in V N(G)$. Consequently $n \odot m=0$ for each $n \in V N(G)^{*}$. In particular $V N(G)^{*}$ cannot have a left identity.

If $G$ is finite, then $A(G)=V N(G)^{*}$. In particular, $V N(G)^{*}$ is commutative. The following shows that the converse of this also holds when $G$ is amenable.

Proposition 3.3. Let $G$ be amenable. Then $V N(G)^{*}$ is commutative if and only if $G$ is finite.

Proof. If $V N(G)^{*}$ is commutative, then $V N(G)$ has a unique topological invariant mean (see [13, Theorem 5.6] and [7, Proposition 5]). Hence $G$ is discrete by [16, Theorem 11]. Also since $G$ is amenable, it follows from [13, Theorem 5.6] 
and [8, Proposition 3] that $V N(G)=U C B(\hat{G})$. In particular, $G$ is finite by [8, Proposition 1 and Theorem 4].

Let $\operatorname{Rad}\left(V N(G)^{*}\right)$ denote the radical of $V N(G)^{*}$.

THEOREM 3.4. The Banach algebra $V N(G)^{*}$ is semisimple if and only if $G$ is finite.

Proof. If $G$ is finite, then $A(G)=V N(G)^{*}$ which is semisimple.

If $V N(G)^{*}$ is semisimple, we first show that $V N(G)$ must have a unique topological invariant mean. In particular $G$ is discrete [16, Theorem 11]. Indeed, if $m_{1}, m_{2}$ are distinct topological invariant means on $V N(G)$, let $J=\{m \in$ $V N(G)^{*} ;\langle m, \lambda(e)\rangle=0$ and $m \odot \phi=m$ for each state $\phi$ in $\left.A(G)\right\}$. Then $m_{1}-m_{2}$ is a nonzero element in $J . J$ is a right ideal of $V N(G)^{*}$. In fact, if $m \in J$, $n \in V N(G)^{*}$, then $\langle m \odot n, \lambda(e)\rangle=\langle m, \lambda(e)\rangle\langle n, \lambda(e)\rangle=0$ by Lemma 3.1(iv). Also if $\phi$ is a state. in $A(G)$, then $(m \odot n) \odot \phi=(m \odot \phi) \odot n=m \odot n$ by Lemma 3.1(i). A similar argument shows that $J$ is a left ideal. Also $J^{2}=\{0\}$. In particular, $J$ is nil and must be included in $\operatorname{Rad}\left(V N(G)^{*}\right)$ (see Corollary in [11, p. 9]). Hence $V N(G)^{*}$ is not semisimple.

If $G$ is not finite, then $U C B(\hat{G})$ is a proper $C^{*}$-subalgebra of $V N(G)([8$, Proposition 1] and [9, Theorem 3]). Let

$$
K=\left\{m \in V N(G)^{*} ;\langle m, x\rangle=0 \text { for each } x \in U C B(\hat{G})\right\} .
$$

Then $K \neq 0$. Also, an application of Lemma 3.1(i) and (ii) shows that $K$ is a left ideal and $K^{2}=\{0\}$. In particular $K \subseteq \operatorname{Rad}\left(V N(G)^{*}\right)$ and hence $V N(G)^{*}$ is not semisimple.

Let $K_{\rho}=\left\{m \in V N(G)^{*} ;\langle m, \lambda(f)\rangle=0\right.$ for each $\left.f \in L_{1}(G)\right\}$.

THEOREM 3.5. $K_{\rho}$ is a weak*-closed two-sided ideal in $V N(G)^{*}$ containing $\operatorname{Rad}\left(V N(G)^{*}\right)$. The quotient Banach algebra $V N(G)^{*} / K_{\rho}$ is isometrically isomorphic to $B_{\rho}(G)$. Also $K_{\rho}=\operatorname{Rad}\left(V N(G)^{*}\right)$ if and only if $G$ is discrete.

Proof. Suppose $n \in V N(G)^{*}$, then $n \odot x \in C_{\rho}^{*}(G)$ for each $x \in C_{\rho}^{*}(G)$ by [13, Proposition 5.2]. Hence $m \odot n \in K_{\rho}$ whenever $m \in K_{\rho}$. Since $K_{\rho}$, being the intersection of weak*-closed sets, is weak*-closed, it follows from Lemma 3.1(iii) that $K_{\rho}$ is an ideal.

Since $K_{\rho}=C_{\rho}^{*}(G)^{\perp}$, the annihilator of $C_{\rho}^{*}(G)$, there is a natural linear isometry $\pi$ from the quotient space $V N(G)^{*} / K_{\rho}$ onto $C_{\rho}^{*}(G)^{*}$. A simple computation shows that $\pi$ is even an algebra isomorphism when $C_{\rho}^{*}(G)^{*}$ is equipped with the induced Arens product. Since the Arens product on $C_{\rho}^{*}(G)^{*}=B_{\rho}(G)$ is precisely the pointwise multiplication, [13, Proposition 5.3], the second assertion follows.

Finally, since $B_{\rho}(G)$ is semisimple, $K_{\rho}$ must contain $\operatorname{Rad}\left(V N(G)^{*}\right)$.

If $G$ is discrete, then $C_{\rho}^{*}(G)=U C B(\hat{G})$ [8, Proposition 3]. Hence if $m \in K_{\rho}$, then Lemma 3.1(i) and (ii) implies that $n \odot m=0$ for all $n \in V N(G)^{*}$. In particular, $K_{\rho}$ is nil, and hence must be included in $\operatorname{Rad}\left(V N(G)^{*}\right)$.

Conversely if $G$ is not discrete, then $1 \notin C_{\rho}^{*}(G)$ by [13, Corollary 4.3]. Choose $n \in V N(G)^{*}$ such that $n(1) \neq 0$ and $n(x)=0$ for all $x \in C_{\rho}^{*}(G)$. If $K_{\rho}=$ $\operatorname{Rad}\left(V N(G)^{*}\right)$, then $n \in \operatorname{Rad}\left(V N(G)^{*}\right)$, which contradicts the second statement of Theorem 3.5 (whose proof does not depend on this result). 
Let $C_{\delta}^{*}(G)$ denote the $C^{*}$-subalgebra of $V N(G)$ generated by the left translations $\{\lambda(g) ; g \in G\}$ on $L_{2}(G)$. Then for each $g \in G, \phi \in A(G), \phi \cdot \lambda(g)=\phi(g) \lambda(g)$. For $n \in V N(G)^{*}, \phi \in A(G)$ and $g \in G$, we have

$$
\langle n \odot \lambda(g), \phi\rangle=\langle n, \phi \cdot \lambda(g)\rangle=\langle n, \lambda(g)\rangle \phi(g)=\langle\langle n, \lambda(g)\rangle \lambda(g), \phi\rangle
$$

which shows that $C_{\delta}^{*}(G)$ is topologically introverted.

Let $B_{\delta}(G)$ denote the linear span of $P_{\delta}(G)$, where $P_{\delta}(G)$ is the pointwise closure of $A(G) \cap P(G)$. Then $B_{\delta}(G)$ is a subalgebra of $B\left(G_{d}\right)$, where $G_{d}$ denotes the group $G$ with the discrete topology. Furthermore, $B_{\delta}(G)$ can be identified with $C_{\delta}^{*}(G)^{*}$ by the map $\pi(\phi)(f)=\Sigma\{\phi(t) f(t) ; t \in G\}$ for each $f \in l_{1}(G)$ and $\phi \in B_{\delta}(G)$ (see [6, Proposition 1.21]). Then, as readily checked, $\pi(\phi \cdot \psi)=\pi(\phi) \odot \pi(\psi)$ for all $\phi, \psi \in$ $B_{\delta}(G)$.

Let $K_{\delta}=\left\{m \in V N(G)^{*} ;\langle m, \lambda(g)\rangle=0\right.$ for all $\left.g \in G\right\}$. Then $K_{\delta}$ is a weak*closed two-sided ideal in $V N(G)^{*}$.

THEOREM 3.6. $B_{\delta}(G)$ with pointwise multiplication and the dual norm is a semisimple commutative Banach algebra isometric algebra isomorphic to $V N(G)^{*} / K_{\delta}$. In particular $K_{\delta}$ contains $\operatorname{Rad}\left(V N(G)^{*}\right)$. The natural embedding $\pi$ of $A(G)$ into $V N(G)^{*} / K_{\delta}$ is a linear isometry. Furthermore, if $G$ is amenable, then $\pi$ is onto if and only if $G$ is finite.

Proof. That $\pi$ is a linear isometry follows from the Kaplansky density theorem. If $G$ is finite then clearly $\pi$ is onto. Conversely, if $G$ is amenable, and $\pi$ is onto, then $A(G)$ can be identified with $C_{\delta}^{*}(G)^{*}$. Since $C_{\delta}^{*}(G)$ has a topological invariant mean [16, Theorem 4], there exists $\phi_{0} \in P(G) \cap A(G)$ such that $\phi \phi_{0}=\phi_{0}$ for all $\phi \in P(G)$. In particular $G$ is discrete [16, Proposition 5]. Hence $A(G)=B_{\delta}(G)=$ $B(G)$ by amenability of $G$. So $G$ must be finite. Proofs of the remaining assertions are clear.

TheOREM 3.7. $G$ is discrete if and only if $A(G)$ is an ideal in $V N(G)^{*}$.

Proof. If $G$ is discrete, then $C_{\rho}^{*}(G)=U C B(\hat{G})$ [8, Proposition 3]. Hence if $\phi \in A(G)$ and $m \in V N(G)^{*}$, then

$$
\begin{aligned}
\langle m \odot \phi, x\rangle & =\langle m, \phi \cdot x\rangle=\langle\psi, \phi \cdot x\rangle \quad \text { for some } \psi \in B_{\rho}(G) \\
& =\langle\psi \cdot \phi, x\rangle
\end{aligned}
$$

for each $x \in V N(G)$. Hence $m \odot_{\phi}=\psi \phi$ which is in $A(G)$. It now follows from Lemma 3.1(i) that $A(G)$ is an ideal in $V N(G)^{*}$.

Conversely if $A(G)$ is an ideal in $V N(G)^{*}$, let $\phi_{0} \in A(G) \cap P_{1}(G)$, where $P_{1}(G)=\{\phi \in P(G) ; \phi(e)=1\}$. Let

$$
K=\left\{m \odot \phi_{0} ; m \in V N(G)^{*}, m \geqslant 0 \text { and }\|m\|=1\right\} .
$$

Then the Banach-Alaoglu Theorem and Lemma 3.1(ii) imply that $K$ is a weakly compact convex subset of $A(G)$. Also, if $\phi \in A(G) \cap P_{1}(G)$, let $T_{\phi}(\psi)=\phi \psi$ for each $\psi \in K$. Then $\left\{T_{\phi} ; \phi \in A(G) \cap P_{1}(G)\right\}$ is a commuting family of continuous affine maps from ( $K$, weak) into ( $K$, weak). An application of the MarkovKakutani fixed point theorem [5, p. 456] shows that there exists $\psi_{0} \in K$ such that 
$T_{\phi}\left(\psi_{0}\right)=\psi_{0}$ for all $\phi \in A(G) \cap P_{1}(G)$. In particular $\psi_{0}$ is a topological invariant mean on $V N(G)$. By [16, Proposition 5], $G$ must be discrete.

4. Maximal regular ideals. Let $R$ be a ring. A left ideal $I$ in $R$ is regular if there exists $u \in R$ such that $x u-x \in I$ for each $x \in R$. As is well known, any proper regular left ideal in $R$ is contained in a maximal proper regular left ideal in $R$ (see $[15$, p. 58]). In this section, we shall study in detail the class of maximal proper left ideals in $V N(G)^{*}$ of a locally compact group $G$.

If $g \in G$, let $I_{g}=\{\phi \in A(G) ; \phi(g)=0\}$ and let $I_{g}^{\#}=\{m \in$ $\left.V N(G)^{*} ;\langle m, \lambda(g)\rangle=0\right\}$. Then $I_{g}^{\#}$, being the kernel of the nonzero multiplicative linear functional $\lambda(g)$ on $V N(G)^{*}$ (Lemma 3.1(iv)), is a maximal weak*-closed ideal in $V N(G)^{*}$ and $I_{g}$ is weak*-dense in $I_{g}^{\#}$. Let $T_{g}=\{\phi \in A(G)$; there exist an open set $V$ containing $g$ and $\phi(x)=0$ for all $x \in V\}$. Also if $V$ is an open set in $G$, let

$$
S_{V}=\cap\left\{I_{g} ; g \notin V\right\} .
$$

Then $T_{g}$ and $S_{V}$ are also ideals in $A(G)$, and $S_{V}$ is closed.

Lemma 4.1. Let $M$ be a maximal regular left ideal in $V N(G)^{*}$ and $g \in G$. Then either $M \supseteq T_{g}$ or there exists an open set $V$ of $G$ containing $g$ such that $M \supseteq S_{V}$.

Proof. If $M$ does not contain $T_{g}$, let $\phi \in T_{g}$ and $\phi \notin M$. There exists an open set $V$ of $G$ containing $g$ such that $\phi \in \cap\left\{I_{x} ; x \in V\right\}$. It follows from the maximality of $M$ that

$$
V N(G)^{*}=M+V N(G)^{*} \odot \phi .
$$

Let $u \in V N(G)^{*}$ such that $n \odot u-n \in M$ for all $n \in V N(G)^{*}$. Then $u=m+$ $p \odot \phi$ for some $p \in V N(G)^{*}$ and $m \in M$. Let $\psi \in S_{V}$, then

$$
\psi \odot u=\psi \odot(m+p \odot \phi)=\psi \odot m+p \odot \psi \phi .
$$

Now $\psi(x)=0$ for all $x \notin V$ by definition of $S_{V}$, and $\phi(x)=0$ if $x \in V$. Hence $(\psi \phi)(x)=\psi(x) \phi(x)=0$ for all $x \in G$. Consequently, $\psi \phi=0$ and so

$$
\psi-\psi \odot u=\psi-\psi \odot m \in M .
$$

In particular $\psi \in M$. Thus $S_{V} \subseteq M$.

Lemma 4.2. Let $g \in G$. Then $I_{g}$ is contained in the norm closure of $T_{g}$.

Proof. This follows directly from Corollary 2(4.11) in [6].

THEOREM 4.3. Every maximal regular left ideal in $V N(G)^{*}$ contains $I_{g}$ for some $g \in G$.

Proof. Let $M$ be a maximal regular left ideal in $V N(G)^{*}$. Then $M$ is closed [15, p. 68]. If $M \supset I_{g}$ for any $g \in G$, then by Lemma $4.2, M \supset T_{g}$ for any $g \in G$. By Lemma 4.1, we have for each $g \in G$ an open set $V(g)$ containing $g$ such that $M \not \partial S_{V(g)}$. Let $J$ denote the smallest (left) ideal in $A(G)$ containing each $S_{V(g)}$. If $J$ is not dense in $A(G)$, then by the Tauberian property of $A(G)$ [6, p. 223], there exists $g_{0} \in G$ such that $J \subseteq I_{g_{0}}$. Consequently, $\phi\left(g_{0}\right)=0$ for all $\phi \in J$. However, by [6, Lemma 3.2], there exists $\phi \in A(G)$ such that $\phi\left(g_{0}\right)=1$ and $\phi \in S_{V\left(g_{0}\right)}$. But 
$S_{V\left(g_{0}\right)} \subseteq J$, which is impossible. Hence $J$ is dense in $A(G)$. Since $M \supseteq S_{V(g)}$ for each $g \in G$, it follows that $M \supseteq J$. But $M$ is closed. Hence $M \supseteq A(G) \supseteq I_{g}$ for all $g \in G$.

COROllary 4.4. Let $M$ be a maximal regular left ideal in $V N(G)^{*}$. Then either $M \supseteq A(G)$ or there exists a unique $g \in G$ such that $M \supseteq I_{g}$.

Proof. By Theorem 4.3, $M \supseteq I_{g}$ for some $g \in G$. Since $M \cap A(G)$ is an ideal in $A(G)$ and the ideals $I_{g}$ are all maximal and distinct in $A(G), A(G) \subseteq M$ if $M$ contains more than one $I_{g}$.

COROllary 4.5. Let $M$ be a maximal regular left ideal in $V N(G)^{*}$. If there exists $x \in V N(G)$ such that $M \subseteq\left\{m \in V N(G)^{*} ; m(x)=0\right\}$, then there exists $g \in G$ such that $M=I_{g}^{\#}$.

Proof. By Theorem 4.3, there exists $g \in G$ such that $M \supseteq I_{g}$. So $\langle\phi, x\rangle=0$ for all $\phi \in I_{g}$. Since $x \neq 0, x=\alpha \lambda(g)$ for some nonzero $\alpha \in \mathbf{C}$. In particular $M=$ $I_{g}^{\#}$.

COROLlary 4.6. All maximal regular left ideals of $V N(G)^{*}$ are weak*-dense except those of the form $I_{g}^{\#}, g \in G$.

Proof. Let $M$ be a maximal left ideal of $V N(G)^{*}$. If the weak*-closure of $M$ is not $V N(G)^{*}$, then there exists $x \in V N(G)$ such that $\langle x, m\rangle=0$ for all $m \in M$, and $x \neq 0$. By Corollary 4.5, $M=I_{g}^{\#}$ for some $g \in G$.

We need the following simple observation.

LEMma 4.7. If $G$ is not discrete, then $\lambda(g) \notin C_{\rho}^{*}(G)$ for any $g \in G$.

Proof. If $\lambda(g) \in C_{\rho}^{*}(G)$, then $\lambda\left(g^{-1}\right) \notin C_{\rho}^{*}(G)$ by [13, Corollary 4.3]. Hence there exists $m \in V N(G)^{*}$ such that $m\left(\lambda\left(g^{-1}\right)\right) \neq 0$ and $m(x)=0$ for each $x \in$ $C_{\rho}^{*}(G)$. Let $n \in V N(G)^{*}$ be defined by $n(x)=\overline{m\left(x^{*}\right)}, x \in V N(G)$. Then $n(\lambda(g))$ $\neq 0$ and $n(x)=0$ for each $x \in C_{\rho}^{*}(G)$, which is impossible.

THEOREM 4.8. $G$ is discrete if and only if each maximal regular left ideal in $V N(G)^{*}$ either contains $A(G)$ or is of the form $I_{g}^{\#}$ for some $g \in G$.

Proof. Assume that $G$ is discrete and that $M$ is a maximal regular left ideal in $V N(G)^{*}$. If $M$ does not contain $A(G)$, then by Corollary 4.4 , there exists a unique $g \in G$ such that $I_{g} \subseteq M$. Since $A(G) \cap M$ is a proper closed ideal in $A(G)$ containing $I_{g}$ and $I_{g}$ is maximal in $A(G)$, we have $A(G) \cap M=I_{g}$. We shall show that $M=I_{g}^{\#}$.

Let $\phi_{0} \in A(G)$ be such that $\phi_{0}(g)=1$ [6, Lemma 3.2]. Then $\phi_{0}^{2}-\phi_{0} \in I_{g}$ and $\phi_{0}^{2} \notin I_{g}$. In particular $\phi_{0}^{2} \notin M$ and $V N(G)^{*}=M+V N(G)^{*} \odot \phi_{0}$ by maximality of $M$. Now if $p \in I_{g}^{\#}$, then $p=m+n \odot \phi_{0}$ for some $m \in M$ and $n \in V N(G)^{*}$. Hence $\phi_{0} \odot p=\phi_{0} \odot m+\phi_{0}^{2} \odot n$. Since $\phi_{0} \odot p \in A(G)$ (by Theorem 3.7) and $\phi_{0} \odot p$ $\in I_{g}^{\#}$, we have $\phi_{0} \odot p \in I_{g}$. So $\phi_{0} \odot p \in M$. Hence $\phi_{0}^{2} \odot n=\phi_{0} \odot p-\phi_{0} \odot m$ is also in $M$. However $\left(\phi_{0}^{2}-\phi_{0}\right) \odot n \in I_{g}^{\#} \cap A(G)=I_{g}$ by Theorem 3.7. Hence $\phi_{0} \odot n \in$ $M$. Consequently $p \in M$. Since $p$ is arbitrary, we have $I_{g}^{\#} \subseteq M$. But $I_{g}^{\#}$ is also maximal, hence $I_{g}^{\#}=M$. 
Conversely if $G$ is not discrete, let $g_{0} \in G$ be fixed. If $m \in V N(G)^{*}$, let $r(m)$ denote the restriction of $m$ to $C_{\rho}^{*}(G)$. Then $r(m)$ may be regarded as an element in $B_{\rho}(G)$. Define $h(m)=r(m)\left(g_{0}\right)$. Since the Arens product on $C_{\rho}^{*}(G)$ is precisely the pointwise multiplication on $B_{\rho}(G)$ [13, Proposition 5.3], it follows that $h$ is a nonzero continuous multiplicative linear functional on $V N(G)$. Hence $M=h^{-1}(0)$ is a maximal regular left ideal in $V N(G)^{*}$. M does not contain $A(G)$ since $h(\psi)=1$ for any $\psi \in A(G)$ such that $\psi\left(g_{0}\right)=1$. Also $M \neq I_{g}^{\#}$ for any $g \in G$. Indeed if $M=I_{g}^{\#}$, then $m(\lambda(g))=0$ for all $m \in M$. But $\lambda(g) \notin C_{\rho}^{*}(G)$ by Lemma 4.7 and so there exists $m \in V N(G)^{*}$ such that $m(\lambda(g)) \neq 0$ and $m(x)=0$ for all $x \in$ $C_{\rho}^{*}(G)$. In particular $r(m)\left(g_{0}\right)=0$ and so $m \in M$, which is impossible.

TheOREM 4.9. Let $G$ be an amenable locally compact group. Then the following statements are equivalent.

(a) $G$ is compact.

(b) Each maximal regular left ideal $M$ in $V N(G)^{*}$ contains $I_{g}$ for some unique $g \in G$.

(c) The right identity of $V N(G)^{*}$ is contained in the left ideal $J$ generated by $A(G)$.

Proof. (a) $\Rightarrow$ (b) If $G$ is compact, then $A(G)$ contains the identity of $V N(G)^{*}$. Hence there exists no proper left ideal of $V N(G)^{*}$ which contains $A(G)$. Apply now Corollary 4.4.

(b) $\Rightarrow$ (c) If the right identity of $V N(G)^{*}$ is not contained in $J$, then there exists a maximal regular left ideal $M$ in $V N(G)^{*}$ containing $J$ (see [15, pp. 58 and 68]). Hence (b) fails.

(c) $\Rightarrow$ (a) Let $\theta$ be the right identity of $V N(G)^{*}$. If $\theta \in J$, then $\theta=\sum_{1}^{n} \phi_{i} \odot m_{i}$, $\phi_{i} \in A(G)$ and $m_{i} \in V N(G)^{*}$. Then for each $\psi \in A(G)$ and $q \in V N(G)^{*}$, we have

$$
\begin{aligned}
\sum_{1}^{n} \psi \phi_{i} \odot\left(q \odot m_{i}-m_{i} \odot q\right) & =q \odot \sum_{1}^{n} \psi \phi_{i} \odot m_{i}-\left(\sum_{1}^{n} \psi \phi_{i} \odot m_{i}\right) \odot q \\
& =q \odot \psi-\psi \odot q=0 .
\end{aligned}
$$

Since $\theta$ is the weak*-limit of a net in $A(G)$, it follows that

$$
\sum_{1}^{n} \theta \odot \phi_{i} \odot\left(q \odot m_{i}-m_{i} \odot q\right)=0
$$

also. Consequently

$$
\begin{aligned}
q-\theta \odot q & =\sum_{1}^{n} q \odot \phi_{i} \odot m_{i}-\sum_{1}^{n} \phi_{i} \odot m_{i} \odot q \\
& =\sum_{1}^{n} \phi_{i} \odot q \odot m_{i}-\sum_{1}^{n} \phi_{i} \odot m_{i} \odot q \\
& =\sum_{1}^{n} \phi_{i} \odot \theta \odot q \odot m_{i}-\sum_{1}^{n} \phi_{i} \odot \theta \odot m_{i} \odot q \\
& =0
\end{aligned}
$$

since $A(G)$ is in the centre of $V N(G)^{*}$. Hence $\theta$ is an identity of $V N(G)^{*}$, which implies that $G$ is compact (Proposition 3.2). 
COROllary 4.10. Let $G$ be an amenable locally compact group. Then $G$ is finite if and only if each maximal regular left ideal of $V N(G)^{*}$ is of the form $I_{g}^{\#}$ for some $g \in G$.

Proof. This follows readily from Theorems 4.8 and 4.9.

5. An isomorphism theorem. We shall prove in this section that if $G_{1}$ and $G_{2}$ are discrete groups, and if the Banach algebras $V N\left(G_{1}\right)^{*}$ and $V N\left(G_{2}\right)^{*}$ are isometric and order isomorphic, then $G_{1}$ and $G_{2}$ are isomorphic. We begin with some observations.

Given any group $G$, the second conjugate algebra $V N(G)^{* *}$ is again a von Neumann algebra.

LEMMA 5.1. Let $G$ be a discrete group and $u$ be an invertible element in $V N(G)^{* *}$. If $u$ is multiplicative on $V N(G)^{*}$, then $u=\lambda(g)$ for some $g \in G$.

Proof. Let $M=\left\{m \in V N(G)^{*} ;\langle u, m\rangle=0\right\}$. Then $M$ is a regular maximal left ideal in $V N(G)^{*}$. If $M=I_{g}^{\#}$, then $u=\alpha \lambda(g)$ for some $\alpha \in \mathbf{C}$. Choose $\phi \in A(G)$ such that $\phi(g)=1$. Then $\alpha=\left\langle u, \phi^{2}\right\rangle=\langle u, \phi\rangle\langle u, \phi\rangle=\alpha^{2}$. So $\alpha=1$, i.e. $u=$ $\lambda(g)$. Otherwise, using Theorem 4.8, $M$ must contain $A(G)$. Let

$$
A(G)^{0}=\left\{f \in V N(G)^{* *} ;\langle f, \phi\rangle=0 \text { for each } \phi \in A(G)\right\} .
$$

Then $A(G)^{0}$ is a weak ${ }^{*}$-closed ideal in $V N(G)^{* *}$ (see [17, p. 44]). Hence there exists $z$, a central projection in $V N(G)^{* *}$, such that $A(G)^{0}=z \cdot V N(G)^{* *}$. Then $z u=u$ and $z=z\left(u u^{-1}\right)=u u^{-1}=e$, where $e$ is the identity in $V N(G)^{* *}$. Hence $A(G)^{0}=$ $V N(G)^{* *}$, which is impossible.

LEMMA 5.2. Let $W_{1}, W_{2}$ be von Neumann algebras with identities $e_{1}, e_{2}$ respectively. Let $\Phi$ be a linear isometry from $W_{1}$ onto $W_{2}$ such that $\Phi\left(e_{1}\right)=e_{2}$. Let $G_{1}$ be a group of invertible elements in $W_{1}$. If $\Phi\left(G_{1}\right)=G_{2}$ is a group of invertible and linearly independent elements in $W_{2}$, then $G_{1}$ and $G_{2}$ are isomorphic as discrete groups.

Proof. We follow an idea of Martin Walter in the proof of Theorem 3 [18, p. 29]. For each $x \in G_{1}$, let

$$
H_{x}=\left\{y \in G_{1} ; \phi(x y)=\phi(x) \phi(y)\right\}, \quad K_{x}=\left\{y \in G_{1} ; \phi(x y)=\phi(y) \phi(x)\right\} .
$$

We shall show that for each $x \in G_{1}$,

(i) $H_{x} \cup K_{x}=G_{1}$,

(ii) $H_{x}$ and $K_{x}$ are subgroups of $G_{1}$.

When this is done, then for each $x \in G$, either $H_{x}=G_{1}$, or $K_{x}=G_{1}$. Let $H=\cap\left\{H_{x} ; x \in G_{1}\right\}$ and $K=\cap\left\{K_{x} ; x \in G_{1}\right\}$. Then $H, K$ are subgroups of $G_{1}$. Also if $x \in G_{1}$ and $H_{x}=G_{1}$, then by [12, Lemma 6],

$$
\Phi(x \cdot y)+\Phi(y \cdot x)=\Phi(x) \Phi(y)+\Phi(y) \Phi(x)
$$

for each $y \in G_{1}$. So $\Phi(y \cdot x)=\Phi(y) \Phi(x)$ and $x \in H$. Similarly if $K_{x}=G_{1}$, then $x \in K$. Consequently $H \cup K=G_{1}$. Hence either $H=G_{1}$ or $K=G_{1}$. In each of the two cases, the groups $G_{1}$ and $G_{2}$ are isomorphic. It remains to prove (i) and (ii).

Let $y \in G_{1}$. If $x y=y x$, then $\Phi(x) \Phi(y)=\Phi(y) \Phi(x)$ by [12, Theorem 5]. Hence $y \in H_{x} \cap K_{x}$ by (1). If $x y \neq y x$ and $y \notin H_{x} \cup K_{x}$, then $\Phi(x y), \Phi(y x), \Phi(x) \Phi(y)$ 
and $\Phi(y) \Phi(x)$ are distinct elements in $G_{2}$. In fact, if $\Phi(x) \Phi(y)=\Phi(y) \Phi(x)$, then applying [12, Theorems 5 and 7] to the operator $\Phi^{-1}$, we get $x y=y x$. Also, $\Phi(y x) \neq \Phi(x) \Phi(y)$. For otherwise $\Phi(x y)=\Phi(y) \Phi(x)$, i.e. $y \in K_{x}$, by (1). Similarly, $\Phi(y x) \neq \Phi(y) \Phi(x)$. Since distinct elements in $G_{2}$ are linearly independent (by assumption), this contradicts (1). Hence (i) holds.

To prove (ii), let $z_{i} \in W_{i}, i=1,2$, be central projections such that $\Phi$ is a *-isomorphism from $W_{1} z_{1}$ onto $W_{2} z_{2}$ and a ${ }^{*}$-anti-isomorphism from $W_{1}\left(e_{1}-z_{1}\right)$ onto $W_{2}\left(e_{2}-z_{2}\right)$ (see [12, Theorem 10]). Then $\Phi\left(x z_{1}\right)=\Phi(x) z_{2}$ and $\Phi\left(x\left(e_{1}-z_{1}\right)\right)$ $=\Phi(x)\left(e_{2}-z_{2}\right)$ for all $x \in W_{1}$. Observe that

$$
y \in K_{x} \text { if and only if }(x y-y x) z_{1}=0
$$

and

$$
y \in H_{x} \text { if and only if }(x y-y x)\left(e_{1}-z_{1}\right)=0 .
$$

To prove (2), let $y \in K_{x}$, then

$$
\begin{aligned}
\Phi\left((x y-y x) z_{1}\right) & =\Phi(x y-y x) z_{2} \\
& =\Phi(y) \Phi(x) z_{2}-\Phi\left(y z_{1}\right) \Phi\left(x z_{1}\right) \\
& =0 .
\end{aligned}
$$

Hence $(x y-y x) z_{1}=0$. Conversely if $(x y-y x) z_{1}=0$ and $y \notin K_{x}$, then $y \in H_{x}$ by (i). Hence $(x y-y x)\left(e_{1}-z_{1}\right)=0$. So $x y=y x$. Consequently $y \in K_{x}$ by [12, Theorem 5] and (1). Statement (3) can be proved similarly. Now if $y_{1}, y_{2} \in K_{x}$, then

$$
x\left(y_{1} y_{2}\right) z_{1}=x y_{1} z_{1} y_{2}=\left(y_{1} x\right) z_{1} y_{2}=y_{1}\left(x y_{2}\right) z_{1}=\left(y_{1} y_{2}\right) x z_{1}
$$

using (2). Hence $y_{1} y_{2} \in K_{x}$ by (2) again. Also if $y \in K_{x}$, then $\left(y^{-1} x-x y^{-1}\right) z=$ $y^{-1}[(y x-x y) z] y^{-1}=0$. Hence $y^{-1} \in K_{x}$ by (2). Hence $K_{x}$ is a subgroup of $G_{1}$. Similarly, $H_{x}$ is also a subgroup of $G_{x}$.

THEOREM 5.3. Let $G_{1}, G_{2}$ be discrete groups. If there exists an order preserving isometric algebra isomorphism from $V N\left(G_{2}\right)^{*}$ onto $V N\left(G_{1}\right)^{*}$, then $G_{1}$ and $G_{2}$ are isomorphic.

Proof. We identify $\lambda(x) \in V N\left(G_{i}\right)$ with $x \in G_{i}, i=1,2$. Let $U$ be an order preserving isometric isomorphism from $V N\left(G_{2}\right)^{*}$ onto $V N\left(G_{1}\right)^{*}$, and $\Phi=U^{*}$. Let $e_{i}$ denote the identity of $G_{i}$. If $n \in V N(G)^{*}, n \geqslant 0$, then $\left\langle n, \Phi\left(e_{1}\right)\right\rangle=\left\langle U(n), e_{1}\right\rangle$ $=\|U(n)\|=\|n\|=\left\langle n, e_{2}\right\rangle$. Hence $\Phi\left(e_{1}\right)=e_{2}$. By [12, Theorem 7 and Lemma 12], $\Phi$ is a Jordan *-isomorphism mapping the set of unitary elements in $V N\left(G_{1}\right)^{* *}$ onto $V N\left(G_{2}\right)^{* *}$. By Lemma $5.1, \Phi$ maps $G_{1}$ onto $G_{2}$. Also, elements in $G_{2}$, being multiplicative on $V N(G)^{*}$, are linearly independent (see [2, Lemma 17 and Corollary 18, p. 93] and note that their proofs do not depend on the fact that the Banach algebra is commutative). The conclusion now follows from Lemma 5.2.

We thank the referee for his many valuable suggestions. Lemma 3.1 is suggested to us by him.

\section{REFERENCES}

1. R. Arens, The adjoint of a bilinear operation, Proc. Amer. Math. Soc. 2 (1951), 839-849.

2. F. F. Bonsall and J. Duncan, Complete normed algebras, Springer-Verlag, Berlin and New York, 1973. 
3. P. Civin, Ideals in the second conjugate algebra of a group algebra, Math. Scand. 11 (1962), $161-174$.

4. P. Civin and B. Yood, The second conjugate space of a Banach algebra as an algebra, Pacific J. Math. 11 (1961), 847-870.

5. N. Dunford and J. T. Schwartz, Linear operators. I, Interscience, New York, 1958.

6. P. Eymard, L'algèbre de Fourier d'un groupe localement compact, Bull. Soc. Math. France 92 (1964), 181-236.

7. E. E. Granirer, Weakly almost periodic and uniformly continuous functionals on the Fourier algebra of any locally compact group, Trans. Amer. Math. Soc. 189 (1974), 371-382.

8. Density theorems for some linear subspaces and some $C^{*}$-subalgebras of $V N(G)$, Sympos. Mat., vol. XXII, Istit. Nazionale di Alta Mat., 1977, pp. 61-70.

9. __ On group representations whose $C^{*}$-algebra is an ideal in its von Neumann algebra (preprint).

10. F. P. Greenleaf, Invariant means on topological groups and their applications, Van Nostrand Math. Studies, no. 16, Van Nostrand Reinhold, New York, 1969.

11. N. Jacobson, Structure of rings, Amer. Math. Soc. Colloq. Publ., vol. 37, Amer. Math. Soc., Providence, R.I., 1956.

12. R. V. Kadison, Isometries of operator algebras, Ann. of Math. (2) 54 (1951), 325-338.

13. A. T. Lau, Uniformly continuous functionals on the Fourier algebra of any locally compact group, Trans. Amer. Math. Soc. 251 (1979), 39-59.

14. H. Leptin, Sur l'algèbre de Fourier d'un groupe localement compact, C. R. Acad. Sci. Paris Sér. A-B 266 (1968), A1180-A1182.

15. L. H. Loomis, An introduction to abstract harmonic analysis, Van Nostrand, Princeton, N.J., 1953.

16. P. F. Renaud, Invariant means on a class of von Neumann algebras, Trans. Amer. Math. Soc. 170 (1972), 285-291.

17. S. Sakai, $C^{*}$-algebras and $W^{*}$-algebras, Springer-Verlag, Berlin and New York, 1971.

18. M. E. Walter, $W^{*}$-algebras and nonabelian harmonic analysis, J. Funct. Anal. 11 (1972), 17-38.

Department of Mathematics, University of Alberta, Edmonton, Alberta, Canada T6G-2G1 